\title{
Analyzing fragmentation of simple fluids with percolation theory
}

\author{
X. Campi*, H. Krivine, N. Sator \\ LPTMS†, F-91405 Orsay Cedex, France \\ and E. Plagnol \\ IPN, F-91406 Orsay Cedex, France
}

June 29, 2021

\begin{abstract}
We show that the size distributions of fragments created by high energy nuclear collisions are remarkably well reproduced within the framework of a parameter free percolation model. We discuss two possible scenarios to explain this agreement and suggest that percolation could be an universal mechanism to explain the fragmentation of simple fluids.
\end{abstract}

PACS. 36.40.Ei Phase transitions in clusters - 36.40.Qv Stability and fragmentation of clusters - 25.70.Mn Nuclear fragmentation

\section{Introduction}

Fragmentation phenomena concern a wide diversity of objects in nature, at many scales of distance and time. A natural question is to ask what is generic and what is specific in these phenomena. Most theoretical efforts made to understand fragmentation apply only to specific objects or, on the contrary, concern simple models with few links with reality [1]. Moreover, experimental data [2 5] are rather sparse and often the experimental conditions are ill defined. As a consequence, the question of the possible existence of universal fragmentation mechanisms remains an open problem [6].

*campi@ipno.in2p3.fr

†UMR 8626, CNRS Université de Paris XI 
The arguments in favour of the existence of universal mechanisms are of various orders. For instance, in aggregation phenomena, which can be considered as the opposite of fragmentation, it is possible to define universal classes [7, 8] (Diffusion Limited Aggregation, Clustering of clusters...) in terms of the initial conditions (number of seeds) and the motion of the aggregating particles (ballistic, Brownian). The fractal structure (dimension) of the aggregation cluster is the fingerprint of these universal classes. In many fragmentation processes the experimental observation, over many orders of magnitude, of power law (scale invariant) fragment size distributions is another possible indication of universal classes. In this case, the value of the power law exponent would be the corresponding fingerprint [2, 6].

Collision induced fragmentation of small fluid drops (atomic nuclei [9], atomic aggregates [10,11], liquid droplets [12]) is a field of experimental research particularly active because it offers the best possibilities of complete identification of the fragmentation products. We present in this paper an analysis of the fragmentation of atomic nuclei in high energy collisions. We show that random percolation theory accounts for experimental data without any adjustable parameter and we discuss two possible explanations for this agreement, depending if one assumes or not that thermal equilibrium is reached before fragmentation occurs. It is then suggested that this percolation type fragmentation mechanism could be universal for simple fluids, i.e. fluids made of structureless particles interacting with short range potentials.

The structure of the paper is the following. In section 2, a brief description of the experiment precedes the analysis of data. Section 3 is devoted to the interpretation of the results. Some final remarks and conclusions are in section 4 .

\section{Analysis of fragmentation data}

\subsection{The experiment}

The experiment was performed by the Aladin collaboration at Gesellschaft für Schwerionenschung (G.S.I.), Darmstadt [13]. Beams of gold ions (Au, $Z=79$ ) at 600 and $1000 \mathrm{MeV} /$ nucleon incident energies were used to bombard targets made of thin copper foils. Data for more than $3.10^{5}$ events were collected. At these high energiest, the commonly admitted scenario of the collision is the following: The part of the projectile that does not geometrically overlap with the target at the point of closest approach is thought to

\footnotetext{
${ }^{1}$ High energies compared with the $8 \mathrm{MeV} /$ nucleon binding energies or the 35 $\mathrm{MeV} /$ nucleon Fermi energies of nuclei.
} 

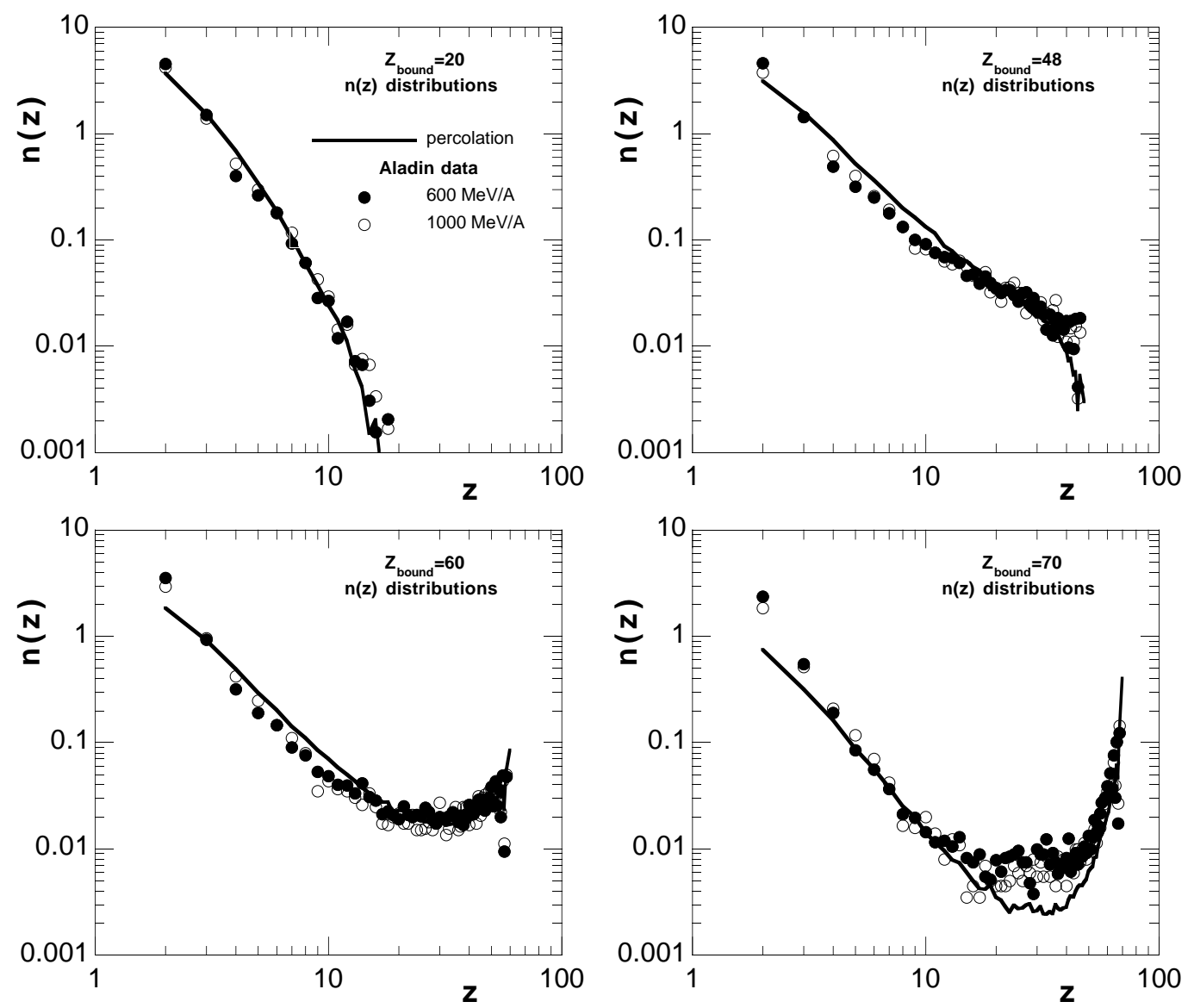

Figure 1: Experimental fragment size distributions $n(z)$ at four values of the control parameter $Z_{\text {bound }}$ (see text). The lines correspond to the percolation calculation. A log-log representation has been chosen to emphasize the power law behaviour at $Z_{\text {bound }}=48$. 


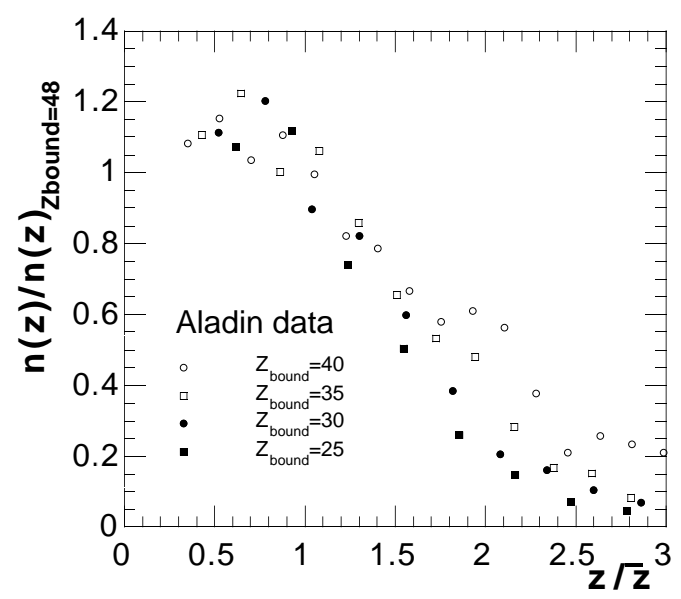

Figure 2: Test of the scaling assumption (Eq. 2), that requires that all the ratios $n(z) / n_{c}(z)$ follow the same curve.

decouple from the rest and form a sub-system called the projectile spectator (PS). The size of this system and its excitation energy $E^{*}$ are therefore dependent on the impact parameter. The overlapping part of the system is completely vaporized and therefore does not contribute to the formation of fragments with nuclear charge $(z)$ greater than one.

In the present experiment, the Aladin device detected with very high efficiency all spectator fragments (those resulting from the decay of the unstable PS) with nuclear charge $z>1$. Neither hydrogen isotopes nor neutrons were detected, event by event, with any significant efficiency. The initial size of the spectator system $\left(Z_{P S}\right)$ is not experimentally measured and can only be inferred from the comparison with a nuclear reaction model. Empirically, this size can be estimated, on average, from the following relation [14]:

$$
<Z_{P S}>=25+Z_{\text {bound }}-0.004 Z_{\text {bound }}^{2}
$$

where $Z_{\text {bound }}$ is equal to the sum of all the charges of products with $z \geq$ 2. In all the studies of these experiments, the parameter $Z_{\text {bound }}$ is used as control parameter. Notice that $Z_{\text {bound }}$, which is closely related to the impact parameter, decreases when the violence of the collision increases.

\subsection{Analysis of data}

To analyze the Aladin data, the following very simple procedure has been used. For a given value of $Z_{\text {bound }}$, the size of the PS was deduced from eq.11 and 

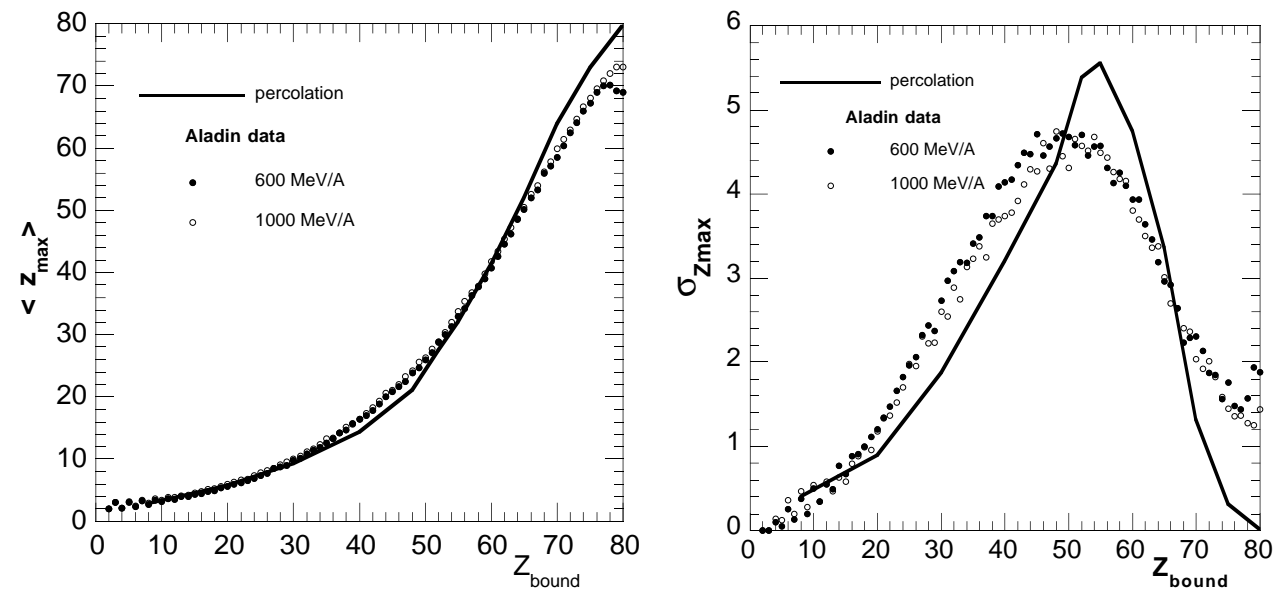

Figure 3: $\quad$ Mean value of $z_{\max }$ as function of $Z_{\text {bound }}$ for percolation calculation and experimental data from ref. 119] (left). Fluctuations of $z_{\max }$ as function of $Z_{\text {bound }}$ for percolation calculation and experimental data from ref. 13] (right).

percolation calculations on a lattice of corresponding size were performed by varying the bond breaking parameter (random-bond percolation). Only those events with the proper $Z_{\text {bound }}$ were kept and the fragment size distribution of these compared to the corresponding experimental data. In this manner the comparison is parameter free. Notice that by fixing a value of $Z_{\text {bound }}$, the maximum value $z_{\max }$ of $z$ is also constrained by this value.

Figure 1 shows, for $Z_{\text {bound }}$ values of 20, 48, 60 and 70, the experimental fragment size distributions. One observes three different regimes of fragmentation. For large $Z_{\text {bound }}$, large impact parameter and low excitation energy, only a heavy residue and light particles are produced. This is the "evaporation" regime. For small $Z_{\text {bound }}$ (most violent collisions) only small fragments are produced and no heavy residue is left. The distribution of this "vaporization" regime decreases exponentially. Remarkably, at $Z_{\text {bound }}$ around 48 an intermediate regime exists, for which the distribution can be fitted by a power law $n(z) \sim z^{-2.2}$. The mean excitation energies per particle can be estimated for the fragmenting systems as a function of $Z_{\text {bound }}$ [14] : they correspond to about $<E^{*}>\simeq 14,6,3,1 \mathrm{MeV}$ per particle for $Z_{\text {bound }}=20,48,60,70$ respectively.

The full lines in figure 1 represent the corresponding distributions obtained from the percolation calculation. The calculation reproduces quanti- 

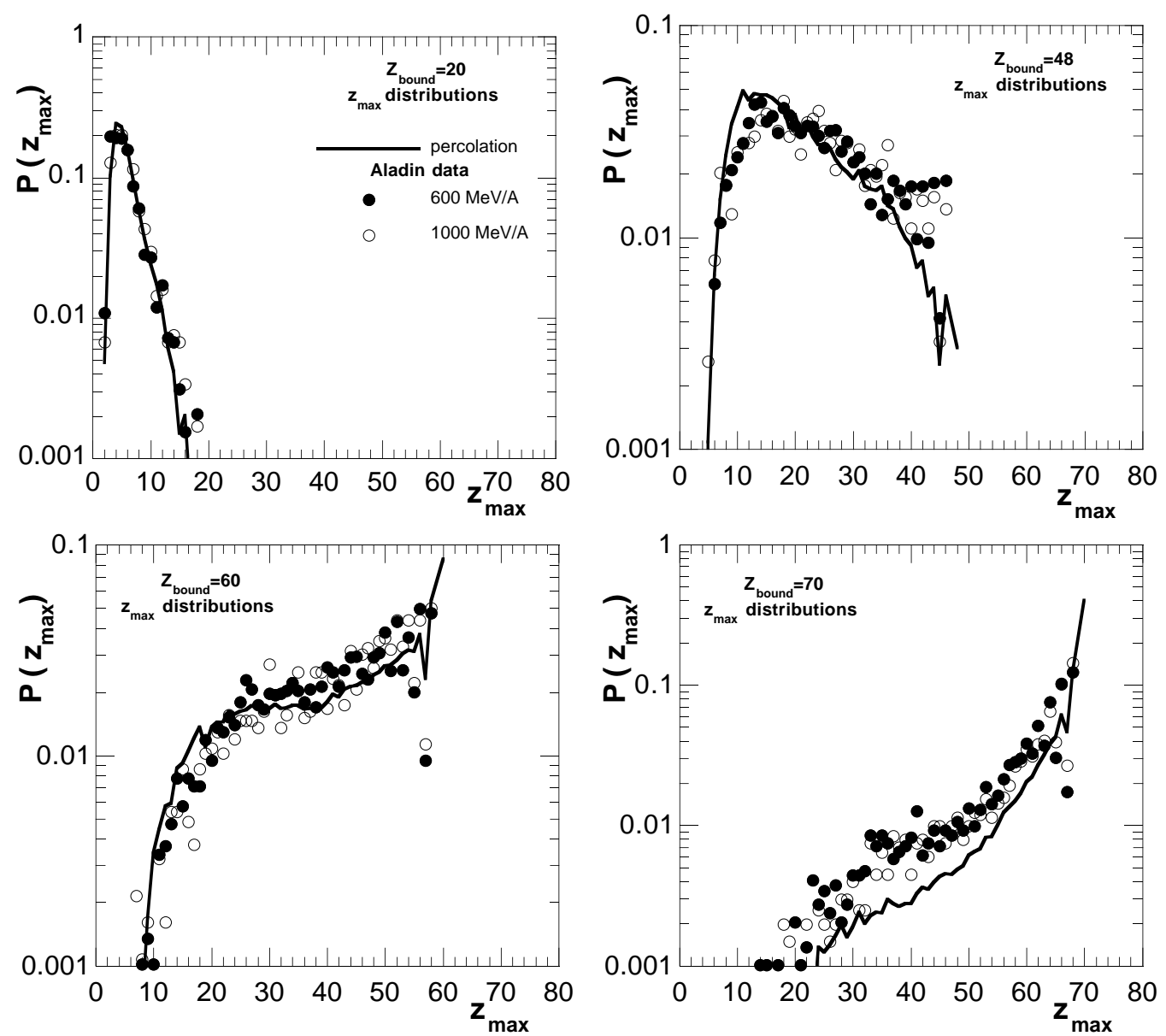

Figure 4: The distribution $P\left(z_{\max }\right)$ of the largest fragment at four values of $Z_{\text {bound. }}$. The circles correspond to the data from ref. 113] and the lines to the percolation calculation. 
tatively, and over several orders of magnitude, the data in the three regimes:2 We are naturally led to associate the experimental power law behaviour at $Z_{\text {bound }} \simeq 48$ to the percolation critical behaviour.

Close to the critical regime, percolation theory predicts typical scaling properties of the cluster size distributions [15]. The size distributions can be reduced to an universal function, $f\left(\frac{z}{\bar{z}}\right)$, by the following formula :

$$
n(z)=n_{c}(z) f\left(\frac{z}{\bar{z}}\right)
$$

where $n_{c}(z)$ is the size distribution at the critical point, taken here as the size distribution observed for $Z_{\text {bound }}=48$. The "characteristic size" $\bar{z}$ is defined by :

$$
\bar{z}=m_{3} / m_{2}
$$

with $m_{k}=\sum_{z \geq 2} z^{k} n^{\prime}(z)$, where $n^{\prime}(z)$ is the mean fragment size distribution obtained excluding, event by event, the largest fragment. One observes in figure 2 that, within the scattering of the data, this rule is rather well satisfied, keeping in mind that this corresponds to the ratio of two quantities varying over more than three orders of magnitude.

The size of the largest fragment plays in percolation theory the role of the order parameter. For an infinite system, it is of infinite size in the percolating phase and finite in the non-percolating one. In a finite system, this transition is smooth, as illustrated in figure 3 . This figure on the left compares, again as a function of $Z_{\text {bound }}$, the experimental measured size of the heaviest fragment $z_{\max }$ to the one obtained from the percolation calculations. The figure on the right shows the fluctuations of $z_{\max }$,

$$
\sigma_{z \max }^{2}=\frac{<z_{\max }^{2}>-<z_{\max }>^{2}}{<z_{\max }>}
$$

As expected [16], these functions show a maximum around the "critical" value of $Z_{\text {bound. }}$. The agreement between experiment and theory is good, except for the highest values $Z_{\text {bound }}$ where the fluctuations should vanish for $z=79$. Very probably, either contributions of the target have been included in the data set or miss-identification of fragments is present. Naturally, the percolation model used here cannot account for either of these features. In figure 4 one can see that even the full distribution $P\left(z_{\max }\right)$ is well accounted for by the calculation.

\footnotetext{
${ }^{2} \mathrm{~A}$ close inspection of the data for $Z_{\text {bound }}=70$ shows an excess of fragments produced around $z=30$ due to the presence of fission events. This is understandably beyond the scope of percolation theory.
} 
By any standard, the agreement observed in figures 11 to 14 , between the experiment and the calculations is very good. In the analysis presented here, as already stressed, no adjustable parameters are used.

\section{Interpretation of the results}

The choice of percolation theory to analyze the experimental results is motivated by the following reasoning. A fully microscopic description of nuclear fragmentation is, a priori, out of scope of theory. Atomic nuclei behave in their ground state as small drops of Fermi liquids composed by particles strongly interacting mainly with a two-body, short range force. This interaction is ill defined at short distances and the techniques used to solve this complicated many body problem are not fully under control. The description of collisions, using transport equations for example, is even more difficult. In view of the above remarks and with the stated goal of understanding the universal features of the data, we believe that a more fruitful point of view is to tackle the problem with the minimum number of assumptions.

The simplest hypothesis would be to assume the equiprobability of all partitions of the integer number $Z=79$. This is equivalent to a "maximum entropy principle" [17, 18]. However, the resulting $n(z)$ are always exponentially decaying functions, in contradiction with experiments.

A step further is to consider topological constraints, by considering fragments in a 3-dimensional space. Among the infinity of models one can imagine to make fragments, random-bond percolation seems a good candidate because of its simplicity and because it retains only the essential constraints. For example, the shape of the fragments is not assumed a priori. It turns out that, as shown in section 2 , it suffices to reproduce very well the experimental data. How does one understand this agreement ? One can imagine at least two scenarios.

In the first scenario, one idealizes the nuclear fluid as an ensemble of particles connected by bonds. The bond between a pair of particles is active as long as the magnitude of their potential energy is greater than the relative kinetic energy. During the collision some of these bonds break because of the change in the position and/or the velocity of the particles. The simplest assumption is that bonds are broken randomly, which corresponds to the usual uncorrelated bond percolation model [15]. Particles connected by unbroken bonds form the fragments. These fragments separate rapidly, pushed away by the long range Coulomb force between protons.

In a second scenario, one assumes that after the collision phase, equilibrium thermodynamics applies. The system expands until a "freezout" 
density is reached, at which the fragments cease to interact by the strong nuclear attractive force and their size distribution is "frozen". The Coulomb force accelerates the fragments, as before. In order to calculate its distribution, we have to define first what a "fragment" is. In the present context, it seems natural to call "fragment" a self-bound ensemble of particles [19,20][. With these definitions, one finds in the $\rho-T$ diagram a percolation line (sometimes called the Kertész line [24]) that separates a percolating and a non-percolating phases. The line joins the thermodynamical critical point to the random bond percolation critical point. On it the fragment size distribution is a power law $n(z) \sim z^{-\tau}$, with $\tau \simeq 2.2$. (see fig. 1 and 2 of ref. [21]). In a small system like an atomic nucleus, rather than a sharp critical line, one finds a "critical zone" on which the $n(z)$ approaches this behaviour (see figure 4 of ref. 25). The similarity of these calculated $n(z)$ with the experimental ones shown in figure 1 is very obvious. Therefore, by inspection of the $n(z)$ alone, it is not possible to disentangle between these two scenarios. However, one could hope that extra information on, for example the fragment kinetic energies, could indicate if thermalization is present or not.

More generally, the main difficulty in analyzing nuclear fragmentation data is due to the very small size of the system, which fundamentally limits the extraction of the universal critical exponents. Indeed, a proper characterization of the physical process requires, apart from the $\tau$ exponent, the determination of the other critical exponents [15] associated with the moments $m_{k}$ of the fragment size distribution. Such measurements are, in principle, possible for simple fluid systems (i.e. made of structureless particles subject to short range forces) of larger sizes such as atomic aggregates or macroscopic pieces of matter, such as liquid drops [12].

We consider as very plausible the possibility to observe this percolation type fragmentation in simple fluids. Indeed, the arguments that we have developed to explain the success of percolation theory should apply without restriction to any system of structureless particles interacting with a short range potential. In fact, attempts have been made to show experimentally this behaviour in the fragmentation of hydrogen aggregates [11]. In these experiments, the multiplicity of fragments $m_{0}$ is used as control parameter. As a function of $m_{0}$ the mass of the largest fragment and its fluctuation, evolve qualitatively as expected in percolation theory. However, in ref. [11], the data are compared with a percolation system of improper size and no definite conclusions can be drawn.

\footnotetext{
${ }^{3}$ Another possibility is to impose the stability by particle evaporation only [21]. At the present level of accuracy, both definitions are operationally very similar. Definition [21] is equivalent to the ones proposed by Hill 22, and by Coniglio and Klein 23.
} 
On the theoretical side, we are performing large scale classical molecular dynamics simulations of a Lennard-Jones fluid, for both the sudden disassembly of an equilibrated system and for the collisions of drops. We clearly find in these calculations the fragment size distributions predicted by percolation theory [26].

\section{Final remarks}

Other experiments of nuclear fragmentation at high bombarding energies have been successfully interpreted with percolation theory [27 30]. However, at lower bombarding energies (30-50 MeV/nucleon) the agreement is less satisfactory [31, 32]. The shape of the $n(z)$ evolves qualitatively as in figure 1 , but a closer examination shows a systematic over-production of intermediate size fragments. The origin of these discrepancies is still unclear. Different explanations can be considered:

a) the identification of the source of the fragments is more difficult at lower collisionnal energies where the separation between the projectile, the target, or the fused system is not as clear as in the case of high energy collisions, leading thus to possible contaminations between these different sub-systems.

b) different fragmentation mechanisms could result from the smaller relative velocities between projectile and target, particularly in the case of fusion at small impact parameter, inducing possible compression effects.

These discrepancies, more generally, could just signal the fact that the assumption that fragmenting nuclei behave as "simple fluids" breaks down at these lower incident energies.

Nuclear fragmentation experiments are often analyzed with the so-called Statistical Multifragmentation Models [33, 34]. In brief, these models deal with the equilibrium thermodynamics of ensembles of spherical drops of nuclear matter confined in a "freezout" volume. Drops interact with each other only by the long range Coulomb force and their internal partition function is taken from empirical mass formulas or from experiment. These modelsf are successful in describing the above mentioned low bombarding energy experiments when fixing the size, the density and the excitation energy of the fragmenting source. For the high energy Aladin data similar analysis have been performed but with a larger set of input parameters [28].

The use of percolation theory concepts is however more comprehensive while much easier to handle. It provides an excellent agreement with the data without requiring any adjustable parameter.

\footnotetext{
${ }^{4}$ These models have been also implemented to study the fragmentation of atomic clusters 35.
} 
We hope that the present results will encourage both the theoretical and the experimental studies of the fragmentation of simple fluids. The fragmentation by collisions of very large aggregates seems particularly promising, because it combines the experimental possibility to detect fragments together with a reduction of finite size and surface corrections effects.

We thank the Aladin collaboration at G.S.I. for allowing us to use their experimental data.

\section{References}

[1] S. Redner, in Statistical models for the fracture of disordered media, ed. H. J. Herrmann and S. Roux (North-Holland, Amsterdam, 1990).

[2] D.L. Turcotte, in Fractals and chaos in geology and geophysics, Cambridge University Press,(1993), and ref. therein.

[3] Fragmentation Phenomena, edited by D. Beyssens et al. (World Scientific, Singapore, 1995).

[4] T. Ishii and M. Matsushita, Jour. of the Phys. Soc. of Japan, Vol. 61, 3474 (1992).

[5] L. Oddershede, P. Dimon and J. Bohr, Phys. Rev. Lett. 71, 3107 (1993).

[6] F. Kun and H. J. Herrmann, Phys. Rev. E59, 2623 (1999).

[7] H. J. Herrmann, Phys. Rep. 136, 153 (1986) .

[8] R. Jullien and R. Botet, in Aggregation and Fractal Aggregates, World Scientific, Singapore (1987).

[9] L.G. Moretto and G.J. Wozniak, Ann. Rev. Nucl. Part. Sci. 43, 379 (1993).

[10] E.E.B. Campbell, T. Raz and R.D. Levine, Chem. Phys. Lett. 253, 261 (1996) .

[11] B. Farizon et al., Phys. Rev. Lett. 81, 4108 (1998).

[12] A. Menchaca-Rocha et al., J. Fluid Mech. 346, 291 (1997).

[13] A Schüttauf et al., Nucl. Phys. A607, 457 (1996). 
[14] X. Campi, H. Krivine and E. Plagnol, Phys. Rev. C50, R2680 (1994).

[15] D. Stauffer and A. Ahrony, Introduction to percolation Theory, Taylor and Francis Ed., London, 1994.

[16] D. Stauffer Z. Phys. B37, 89 (1980).

[17] J. Aichelin, J. Hüfner and R. Ibarra, Phys. Rev. C30, 107 (1984).

[18] R. Engelman, Phil. Mag. B50, 307 (1984).

[19] C. Dorso and J. Randrup, Phys. Lett. B301, 328 (1993).

[20] A. Puente, Phys. Let. A260, 234 (1999).

[21] X. Campi, H. Krivine and A. Puente, Physica A262, 328 (1999).

[22] T.L. Hill, J. Chem. Phys. 23, 617 (1953).

[23] A. Coniglio and W. Klein, J. Phys A13, 2775 (1980).

[24] J. Kertész, Physica A161, 58 (1989).

[25] X. Campi and H. Krivine, Nucl. Phys. A620, 46 (1997).

[26] X. Campi, H. Krivine and N. Sator, to be published.

[27] X. Campi, J. Phys. A19, L917 (1986) ; X. Campi, Phys. Lett. B208, 351 (1988).

[28] P. Kreutz et al., Nucl. Phys. A556, 672 (1993).

[29] M.L. Gilkes et al., Phys. Rev. Lett. 75, 768 (1995).

[30] W. Bauer and A. Botvina, Phys. Rev. C55, 546 (1997).

[31] N. Marie et al., Phys. Lett. B391, 15 (1997).

[32] M. D'Agostino et al., Nucl. Phys. A650, 329 (1999).

[33] D.H.E. Gross, Phys. Rep. 279, 119 (1997).

[34] J.P. Bondorf et al., Phys. Rep. 257, 133 (1995).

[35] D.H.E. Gross and P.A. Hervieux, Z. Phys. D35, 35 (1995). 